\title{
Mapeamento relacional entre padrões de metadados educacionais
}

Luciana Maria Vieira Pöttker

Doutora em Ciência da InformaçãoUNESP/Marília. Docente do Instituto Federal do Paraná - campus Londrina

Edberto Ferneda

Pós-doutor pela Universidade Federal da Paraíba. Doutor em Ciências da Comunicação (Ciência da Informação) pela Universidade de São Paulo. Docente do Departamento de Ciência da Informação da Universidade Estadual Paulista 'Júlio Mesquita Filho' (UNESP) - Campus Marília.

José Antonio Moreiro-González

Catedrático de Biblioteconomia e Documentação e atual Defensor Universitário da Universidad Carlos III de Madrid (UC3M). Pós- doutor pela Universidade de São Paulo - USP. Doutor pela Universidad Nacional de Educación à Distancia UNED - Espanha.

http://dx.doi.org/10.1590/1981-5344/2843

A diversidade de formatos utilizados para o desenvolvimento de objetos de aprendizagem contribui para que o processo de recuperação de informação relevante permaneça sendo um grande desafio para a Ciência da Informação e a Ciência da Computação. A adoção de metadados para a catalogação destes recursos vem sendo utilizada tanto em âmbito nacional quanto internacional. No entanto, não existe um consenso sobre qual é o melhor padrão de metadados. Tem como objetivo apresentar um mapeamento relacional entre principais padrões de metadados utilizados pelos repositórios de objetos de aprendizagem. A metodologia utilizada se fundamenta em análise exploratória e analítica de repositórios de objetos de aprendizagem para identificar os metadados de cada iniciativa e propor um modelo de 
correlação entre os padrões. Os resultados apontam que os padrões Dublin Core e LOM são referências para outros padrões de metadados. Assim, o mapeamento foi realizado a partir de uma correspondência entre esses padrões e para a validação foram analisados 140 objetos de aprendizagem disponíveis em repositórios na Web. Conclui-se que é possível estabelecer um modelo de correlação entre os metadados com um número significativo de elementos para colaborar com o processo de indexação automática e aprimorar a recuperação de informação.

Palavras-chave: Metadados. Catalogação. Mapeamento. Indexação Automática. Objetos de Aprendizagem.

\section{Crosswalk between educational metadata standards}

The formats diversity used for the development of learning objects contributes to the relevant information retrieval process remains a major challenge for Information Science and Computer Science. The adoption of metadata for cataloging these resources are being used both nationally and internationally. However, there is no consensus regarding the best metadata standard. It aims to present a crosswalk between main metadata standards used by learning objects repositories. Methodology is based on exploratory and analytical analysis of learning object repositories to identify the metadata of each initiative and propose a correlation model between the patterns. The results indicate that Dublin Core and LOM standards are references to other metadata standards. Thus, mapping was performed based on a correspondence between these patterns and validating was analyzed 140 learning objects available in repositories web. It follows that it is possible to establish a correlation model between the metadata with a significant number of elements to collaborate with the automatic indexing process and improve information retrieval.

Keywords: Metadata. Cataloging. Crosswalk. Automatic Indexing. Learning Objects.

Recebido em 15.06.2016 Aceito em 21.06.2018 


\section{Introdução}

O domínio de objetos de aprendizagem (OA) é volumoso, pois é impossível dimensionar a quantidade desses recursos em repositórios ou iniciativas individuais que disponibilizam esses recursos na Web e dinâmico uma vez que novos objetos podem ser acrescentados, excluídos e alterados rapidamente. O processo de recuperação desses recursos é uma tarefa complexa visto que seu acervo é distribuído e composto por uma multiplicidade de formatos muito ampla.

Segundo o Learning Technology Standards Committee Learning Object Metadata (IEEE LTSC LOM, 2002), os objetos de aprendizagem são considerados como sendo qualquer entidade - que pode ser utilizada, reutilizada ou referenciada durante todo o processo de ensino e aprendizagem apoiado por computador.

Um objeto de aprendizagem também pode ser considerado como uma parte digital do material educacional que aborda um tópico claramente identificável ou o resultado da aprendizagem e que tenha o potencial de reutilização em diferentes contextos pedagógicos (WELLER; PEGLER; MASON, 2003), ou seja, os objetos de aprendizagem devem ser concebidos de tal forma que contenham desde aspectos técnicos até um perfil pedagógico bem definido.

Repositórios de Objetos de Aprendizagem (ROA) são bibliotecas digitais que armazenam principalmente material educacional com 0 propósito de permitir o compartilhamento e o reuso desses recursos. Os ROA se diferenciam de outros tipos de repositórios digitais uma vez que agrupam diferentes tipos objetos de aprendizagem que têm como característica a reutilização em diferentes contextos de aprendizagem (TAROUCO; RODRIGUES; SCHMITT, 2013).

Portanto, os repositórios de objetos de aprendizagem são ambientes digitais que têm como propósito disponibilizar recursos educacionais previamente catalogados em um padrão de metadados específico a fim de facilitar a busca e o acesso a esses objetos, bem como garantir sua interoperabilidade e reuso.

Os metadados são uma recomendação da Web Semântica para descrever características relevantes dos recursos informacionais disponíveis na Web. A ideia fundamental da Web Semântica é que os computadores utilizando padrões reconhecidos internacionalmente possam recuperar conteúdos informacionais de maneira mais precisa e significativa, por meio de agentes computacionais (BERNERS-LEE; HENDER; LASSILA, 2001).

Segundo Santos e Alves (2009):

[...] as tecnologias da Web Semântica convergem para a área de Ciência da Informação, estabelecendo uma estreita relação na questão da representação do conhecimento, principalmente no que se refere ao uso de metadados considerados essenciais no 
estabelecimento dos requisitos para uma boa representação dos recursos informacionais na rede.

Corroborando com essa visão, Ferneda (2012, p. 137) aponta que é necessário que as tecnologias da Web Semântica permitam realizar uma descrição formal dos recursos informacionais para "que possam ser utilizadas pelos agentes (robôs) de indexação dos mecanismos de busca".

Assim, a catalogação dos OA permite que o conteúdo educacional seja descrito por meio de refinamentos de elementos ou esquemas de codificação, visando facilitar a localização dos objetos de aprendizagem para possibilitar distribuir os conteúdos de aprendizagem às pessoas certas no tempo certo.

Silva e Souza (2013) enfatizam que os metadados podem ser utilizados tanto para descrever características técnicas de baixo nível (data da criação, por exemplo) quanto para representar atributos de alto nível (grau de dificuldade do recurso educacional, por exemplo). López Guzmán (2005) enfatiza a utilidade da adoção de metadados para descrever um recurso, especialmente quando estes não são textuais, ou seja, quando seu conteúdo não pode ser indexado automaticamente pelos motores de busca, como os recursos multimídia e de áudio, por exemplo.

Dessa forma, o processo de indexação automática tende a ser facilitado por meio do uso de um padrão de metadados e está diretamente relacionado com a eficiência do sistema de recuperação de informação. De acordo com Gil Leiva e Fujita (2012, p. 13), existe uma relação estreita entre a indexação e a recuperação da informação, ou seja, pode-se afirmar que a indexação apresenta características que geram efeitos diretos na eficiência de um sistema de recuperação de informação.

Diante do exposto, essa investigação tem como objetivo apresentar um mapeamento relacional dos principais padrões de metadados educacionais adotados pelos ROA para permitir a indexação automática desses recursos informacionais e facilitar a recuperação de informação relevante.

\section{Metodologia de pesquisa}

Enfatizada na abordagem do problema, essa investigação é classificada como qualitativa, pois se fundamenta em uma análise exploratória e analítica de repositórios de objetos de aprendizagem com o intuito projetar um mapeamento relacional entre os padrões de metadados educacionais adotados para catalogação desses recursos.

As etapas para a realização da investigação envolvem a identificação dos padrões de metadados que tem apoiado a catalogação dos objetos de aprendizagem nos ROA. Posteriormente, realiza-se uma análise dos elementos de refinamento utilizados por cada repositório para a catalogação dos OA, identificando possíveis equivalências entre os elementos.

Por fim, é analisada uma amostra de 140 objetos de aprendizagem (do acervo de repositórios de objetos de aprendizagem investigados) com 
a intenção de identificar os elementos adotados na descrição informacional do recurso para finalmente propor um esquema de mapeamento relacional entre os padrões que servirá como base para uma futura indexação automática dos objetos de aprendizagem.

\section{Padrões de metadados educacionais}

Os padrões de metadados têm se destacado no âmbito da sociedade da informação, pois com sua adoção é possível catalogar, gerenciar e recuperar diferentes domínios de informações com o intuito de torná-las interoperáveis para facilitar o processo de busca e recuperação de informação.

O termo metadados é utilizado de várias formas, em diferentes áreas. Para a Ciência da Informação e a Ciência da Computação é considerado como uma descrição de dados que podem ser utilizados para identificar e descrever características comuns entre diferentes recursos informacionais com a finalidade de facilitar a interoperabilidade e a recuperação na Web.

A National Information Standards Organization (NISO, 2004) define os metadados como informações estruturadas que descrevem, explicam, localizam e facilitam o processo de recuperar, usar ou gerenciar um recurso informacional.

Na concepção de Meadow et al. (2007, p. 43) os metadados são a descrição da organização ou conteúdo de um corpus documental tal como um registro ou um banco de dados, ou seja, se refere ao dado sobre o dado ou a informação sobre a informação. Assim, um registro de metadados tipicamente consiste de um conjunto de elementos (campos) que descrevem semanticamente o conteúdo de um determinado recurso, como por exemplo, o direito de propriedade intelectual, a data de criação, entre outros.

Segundo Lorist e van der Meer (2001), os padrões de metadados garantem que os recursos informacionais sejam compreendidos tanto por pessoas quanto por máquinas e incrementam a interoperabilidade, a interdisciplinariedade e a independência de plataforma.

De acordo com Rehak e Mason (2003) os metadados são essenciais para o processo de recuperação dos objetos de aprendizagem, pois são entidades digitais autossuficientes e reutilizáveis. Esses recursos devem ter uma estrutura externa de informações, os metadados, para facilitar sua identificação, armazenamento e recuperação. Portanto, no escopo desse trabalho estão os padrões de metadados que descrevem recursos educacionais.

Diante desse cenário, essa seção apresenta os fundamentos teóricos de alguns dos padrões de metadados adotados pelos repositórios de objetos de aprendizagem investigados nesse trabalho.

\subsection{Dublin Core (DC)}


O Dublin Core consiste em um dos padrões de metadados para representação de um conjunto de elementos descritivos com a finalidade de facilitar a catalogação de recursos digitais distribuídos pela web. Apresenta um formato menos estruturado e mais flexível, pois adota a sintaxe do Resource Description Framework (RDF) que é uma recomendação da Web Semântica.

Foi desenvolvido por uma iniciativa da Dublin Core Metadata Initiative (DCMI), em 1995, com o objetivo de garantir os princípios de simplicidade, extensibilidade, modularidade e interoperabilidade semântica (DCMI, 2016).

Souza, Vendrusculo e Melo (2000) enfatizam que as principais características do padrão Dublin Core são: a simplicidade para a descrição dos recursos informacionais; a compreensão semântica universal dos elementos; sua dimensão internacional e a extensibilidade.

O DC é composto, originalmente, por 15 elementos que podem ser divididos em três categorias, conforme pode ser visualizado na Figura 1. Os elementos do padrão DC são todos opcionais e repetíveis. Qualquer elemento pode ser modificado por um qualificador.

Figura 1 - Escopo do padrão DC com os elementos agrupados por categoria de metadados

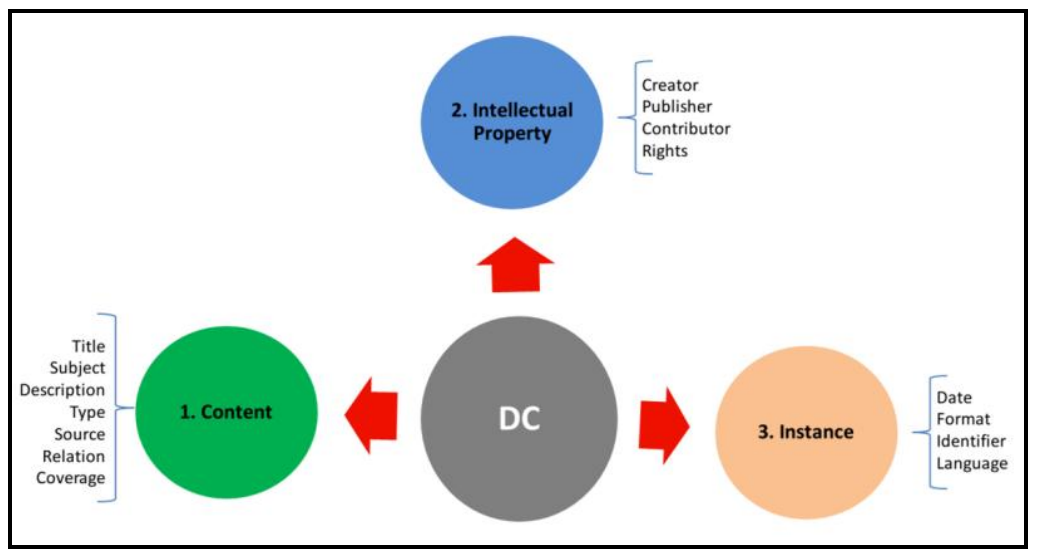

Fonte: Elaboração própria, com base no padrão DC.

Assim, o padrão de metadados DC pode ser utilizado para várias finalidades, desde uma simples descrição de recursos (vídeo, som, imagem, texto e páginas web) até uma combinação de vocabulários de diferentes padrões de metadados, para proporcionar interoperabilidade.

\subsection{Learning Object Metadata (LOM)}

O padrão LOM foi definido pelo Institute of Electrical and Electronic Engineers (IEEE), uma organização internacional que desenvolve padrões e recomendações técnicas para várias áreas de conhecimento. Em 2002, 0 Learning Technology Standards Committee (IEEE LTSC) reconheceu o padrão IEEE 1484.12.2 LOM com o intuito de descrever e classificar objetos de aprendizagem, para que os mesmos possam ser catalogados e 
localizados por motores de busca para serem utilizados e reutilizados em diferentes contextos de aprendizagem (IEEE LTSC LOM, 2002).

A estrutura básica do padrão de metadados educacional LOM V1.0 é apresentada na Figura 2, com a divisão dos elementos classificados nas nove categorias pertencentes ao padrão.

Figura 2 - Escopo do padrão LOM com os elementos agrupados por categoria de metadados

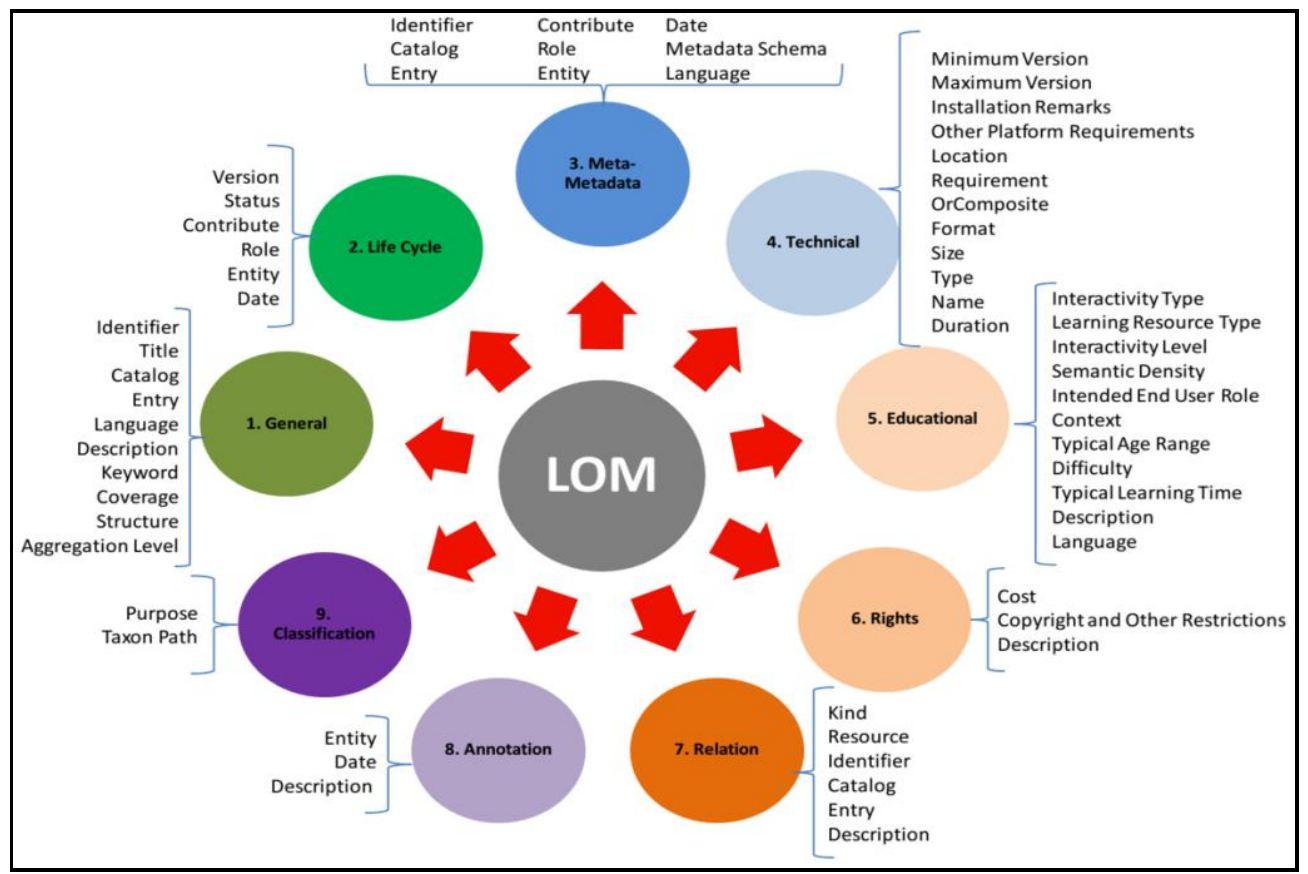

Fonte: Elaboração própria, com base no Schema do LOM V1.0.

De acordo com a IEEE LTSC LOM (2002), a estrutura básica do padrão LOM para descrição de objetos de aprendizagem, ocorre através de campos que estão agrupados por categorias e que podem ser recuperados por qualquer sistema de classificação.

O padrão LOM especifica um cabeçalho que fornece informações sobre o objeto de aprendizagem. Os elementos que compõem esse cabeçalho são os metadados que descrevem semanticamente o objeto de aprendizagem. Assim, esse padrão não interfere no conteúdo ou nas regras dos objetos de aprendizagem, uma vez que apenas agrupa metadados.

Dessa forma, evidencia-se que o padrão LOM tem por objetivo facilitar a indexação, catalogação e busca de objetos de aprendizagem. Esta norma descreve instâncias de metadados para objetos de aprendizagem que representam as características relevantes de como esses recursos podem ser usados, reusados e categorizados.

\subsection{Objetos de Aprendizagem Baseado em Agentes (OBAA)}

O padrão de metadados OBAA é um projeto nacional para objetos de aprendizagem compatível com o padrão LOM que permite a 
interoperabilidade desses objetos nas plataformas Web, TV Digital e para dispositivos móveis, além de suportar requisitos de acessibilidade para pessoas com necessidades especiais e registrar informações educacionais específicas do contexto brasileiro. Também apresenta um modelo básico para a sintaxe e a semântica dos metadados, através da definição de uma ontologia OWL para os OA (VICCARI et al., 2009).

Segundo Viccari et al. (2010), o projeto de OBAA foi desenvolvido com o objetivo de estabelecer uma especificação padronizada para os requisitos técnicos e funcionais de uma plataforma de produção, edição e distribuição de conteúdos digitais interativos, como os objetos de aprendizagem.

O padrão OBAA é uma extensão do padrão LOM, ou seja, o conjunto completo de metadados do OBAA é formado por todas as categorias do LOM, com alguns elementos de metadados adicionais que complementam as categorias Técnica (Technical) e Educacional (Educational); além apresentar duas categorias novas relativas a aspectos de acessibilidade e segmentação.

\section{Correlação dos padrões de metadados educacionais}

No sentido de obter uma correlação entre os padrões de metadados educacionais, as organizações e grupos de pesquisa colaboram para a criação de um padrão universal. Enquanto essa situação não se concretiza, demonstra-se nessa seção um mapeamento entre os principais padrões de metadados educacionais que são adotados pelos ROA avaliados nessa investigação. O Quadro 1 apresenta, de forma breve, uma relação das principais características dos ROA investigados nesse trabalho.

Quadro 1 - Características dos repositórios de objetos de aprendizagem investigados

\begin{tabular}{l|l|l|l}
\hline \multicolumn{1}{c|}{ Repositório } & \multicolumn{1}{c|}{ Disponivel em: } & \multicolumn{1}{c}{$\begin{array}{c}\text { Padrão de } \\
\text { Metadados }\end{array}$} & Arquitetura \\
\hline $\begin{array}{l}\text { BIOE - Banco Internacional de } \\
\text { Objetos de Aprendizagem }\end{array}$ & objetoseducacionais2.mec.gov.br/ & Dublin Core (DC) & $\begin{array}{l}\text { Objetos de aprendizagem e } \\
\text { metadados centralizados }\end{array}$ \\
\hline $\begin{array}{l}\text { CESTA - Coletânea de Entidades } \\
\text { de Suporte ao Uso da Tecnologia } \\
\text { na Aprendizagem }\end{array}$ & $\begin{array}{l}\text { Versão atual (não acessivel): } \\
\text { http://cesta2.cinted.ufrgs.br/xmlui } \\
\text { Versão antiga (funcional): } \\
\text { http://cesta.cinted.ufrgs.br/cesta.login.php }\end{array}$ & $\begin{array}{l}\text { Compativel com o } \\
\text { padrão LOM }\end{array}$ & $\begin{array}{l}\text { Objetos de aprendizagem } \\
\text { distribuidos com indexação } \\
\text { centralizada }\end{array}$ \\
\hline $\begin{array}{l}\text { LUME - Repositório Digital da } \\
\text { Universidade Federal do Rio } \\
\text { Grande do Sul }\end{array}$ & www.lume.ufrgs.br & Dublin Core (DC) & $\begin{array}{l}\text { Objetos de aprendizagem e } \\
\text { metadados centralizados }\end{array}$ \\
\hline $\begin{array}{l}\text { MERLOT II - Multimedia } \\
\text { Education Resource for Learning } \\
\text { and Online Teaching }\end{array}$ & www.merlot.org/merlot/index.htm & LOM & $\begin{array}{l}\text { Objetos de aprendizagem } \\
\text { distribuidos com indexação } \\
\text { centralizada }\end{array}$ \\
\hline $\begin{array}{l}\text { Portal OBAA } \\
\text { ind }\end{array}$ & http://repositorio.portalobaa.org/ & $\begin{array}{l}\text { OBAA compatível } \\
\text { com LOM }\end{array}$ & $\begin{array}{l}\text { Objetos de aprendizagem e } \\
\text { metadados centralizados }\end{array}$ \\
\hline
\end{tabular}

Fonte: Elaboração própria.

O estabelecimento de uma correlação entre os metadados educacionais é importante, pois permite determinar uma associação entre os padrões para facilitar o processo de indexação automática e a posterior recuperação de informação. 
Observou-se que os padrões de metadados mais comumente adotados nos repositórios foram: Dublin Core, LOM e OBAA. Cabe ressaltar que o padrão OBAA é compatível com o LOM. Diante do exposto, optou-se em adotar um padrão de mapeamento entre os elementos do Dublin Core (Figura 1) e LOM (Figura 2) que será utilizado como base para a indexação automática dos objetos de aprendizagem catalogados nos principais repositórios.

Dessa forma, a correlação foi estabelecida tendo como base os quinze elementos padrão Dubin Core, pois conforme enfatizam Silva e Souza (2013), este é o padrão adotado como suplemento de métodos já consolidados para pesquisa e indexação de metadados para Web, uma vez que permite a descrição de qualquer tipo de recurso informacional (textual e multimídia). O Quadro 2 apresenta o mapeamento relacional inicial entre os padrões Dublin Core e LOM.

Quadro 2 - Mapeamento relacional entre os padrões de metadados DC e LOM

\begin{tabular}{l|l}
\hline \multicolumn{1}{c|}{ Dublin Core -DC } & \multicolumn{1}{c}{ LOM } \\
\hline dc.title & general.title \\
\hline dc.subject & general.keyword \\
\hline dc.description & general.description \\
\hline dc.type & educational.learningresourcetype \\
\hline dc.source & technical.location \\
\hline dc.relation & relation.resource \\
\hline dc.coverage & general.coverage \\
\hline dc.creator & lifecycle.entity \\
\hline dc.publisher & lifecycle.role \\
\hline dc.contributor & lifecycle.contribute \\
\hline dc.rights & rights.description \\
\hline dc.date & lifecycle.date \\
\hline dc.format & technical.format \\
\hline dc. identifier & general.identifier \\
\hline dc.language & general.language \\
\hline
\end{tabular}

Fonte: Elaboração própria.

Essa correlação foi obtida pela análise do nível de similaridade entre os elementos dos padrões. Após essa correlação inicial, realizou-se uma verificação na forma como os objetos de aprendizagem estão sendo catalogados nos repositórios de objetos de aprendizagem com a intenção de verificar possíveis equivalências e persistências adicionais que estão sendo aplicadas no processo de catalogação.

O primeiro ROA avaliado foi o Banco Internacional de Objetos de Aprendizagem (BIOE) que adota o padrão Dublin Core. O repositório BIOE apresenta seu acervo dividido em seis categorias principais (Educação Infantil, Ensino Fundamental, Ensino Médio, Educação Profissional, Educação Superior e Modalidades de Ensino) que por sua vez se subdividem em outras 43 categorias, sendo que três delas estão vazias. Neste ROA fazem parte do acervo objetos de aprendizagem nas línguas: portuguesa $(p t)$, inglesa (en) e espanhola (es). Cabe ressaltar que as últimas inserções no repositório datam do ano de 2013. 
Do universo de objetos de aprendizagem constantes em cada categoria, foi selecionando o primeiro recurso de cada subcategoria para avaliação da catalogação do OA. Assim, foram analisados os metadados de 40 recursos educacionais e com isso foi possível verificar a consistência de descrição dos objetos de aprendizagem com o padrão metadados.

O próximo repositório avaliado foi o ROA LUME que apresenta seis categorias (Archives, UFRGS' Events, Scientific Production, Educational Resources, Theses and Dissertations, Academic and Technical Works), subdivididas em outras 27 categorias. Portanto, no escopo deste repositório foram avaliados 27 recursos educacionais. Ressalta-se que 0 procedimento de busca no ROA LUME foi mais complexo, pois esse não apresenta uma relação dos objetos constantes em cada categoria, como observado no repositório BIOE. Assim, utilizou-se como critério de busca palavras-chave aleatórias, mas relacionadas com a temática de cada categoria para selecionar o primeiro recurso recuperado em cada categoria e consequentemente realizar a análise dos OA.

O Quadro 3 apresenta uma equivalência para os elementos de refinamento do padrão de metadados Dublin Core, com base na catalogação dos objetos de aprendizagem do acervo dos ROA: BIOE e LUME. Observou-se que o repositório LUME utiliza alguns elementos de refinamento próprios que estão destacados no Quadro 3.

Quadro 3 - Refinamento na catalogação dos objetos de aprendizagem padrão Dublin Core

\begin{tabular}{|c|c|}
\hline DC & DC - Elemento de refinamento \\
\hline dc.title & dc.title.alternative \\
\hline dc.subject & $\begin{array}{l}\text { dc.subject.keyword } \\
\text { dc.subject.category }\end{array}$ \\
\hline de.description & $\begin{array}{l}\text { dc.description.abstract } \\
\text { dc.description.origem* } \\
\text { dc.description.origin } \\
\text { dc.description.vinculoufrgs* }\end{array}$ \\
\hline dc.type & $\begin{array}{l}\text { dc.type.qualificationlevel } \\
\text { dc.type.qualificationname } \\
\text { dc.type.work }\end{array}$ \\
\hline \multicolumn{2}{|l|}{ dc.source } \\
\hline dc.relation & $\begin{array}{l}\text { dc.relation.isreferencedby } \\
\text { dc.relation.requires }\end{array}$ \\
\hline dc.coverage & $\begin{array}{l}\text { dc.coverage.culture } \\
\text { dc.coverage.temporal }\end{array}$ \\
\hline \multicolumn{2}{|l|}{ dc.creator } \\
\hline dc.publisher & dc.publisher.place \\
\hline dc.contributor & $\begin{array}{l}\text { dc.contributor.advisor } \\
\text { dc.contributor.author } \\
\text { dc.contributor.role } \\
\text { dc.contributor.technicaldirector }\end{array}$ \\
\hline de.rights & dc.rights.license \\
\hline dc.date & $\begin{array}{l}\text { dc.date.accessioned } \\
\text { dc.date.available } \\
\text { dc.date.created } \\
\text { dc.date.issued } \\
\text { dc.date.submitted }\end{array}$ \\
\hline de.format & $\begin{array}{l}\text { dc.format.duration } \\
\text { dc.format.extent } \\
\text { dc.format.original } \\
\text { dc.format.support }\end{array}$ \\
\hline dc.identifier & $\begin{array}{l}\text { dc.identifier.doi } \\
\text { dc.identifier.uri }\end{array}$ \\
\hline dc.language & dc.language.iso \\
\hline
\end{tabular}

Fonte: Elaboração própria.

* elementos identificados apenas no ROA LUME.

O mesmo procedimento adotado para obter a consistência do padrão DC pelos ROA foi realizado para o padrão LOM. Para tanto, foram analisados os repositórios: Portal OBAA, CESTA e MERLOT II. 
O repositório do Portal OBAA possui um acervo de 59 objetos de aprendizagem. Cabe ressaltar que todos os registros de metadados desse acervo estão vazios. Portanto, tomou-se como base para a avaliação os cinco exemplos disponíveis no site no projeto OBBA ${ }^{1}$.

Já o repositório CESTA disponibiliza duas versões de seu repositório: a antiga (que ainda é funcional) e a atual (que não está acessível). O motor de busca desse ROA não permite identificar as categorias dos objetos de aprendizagem, bem como suas subdivisões. Para esse repositório, em específico, não foi avaliado o perfil de catalogação dos OA, uma vez que o ROA disponibiliza um modelo denominado CESTAcore com todos os elementos de refinamento do padrão assim, estabeleceu-se a compatibilidade com o padrão LOM a partir desse modelo.

O repositório Merlot II apresenta seu acervo dividido em nove categorias principais (Academic Support Services, Arts, Business, Education, Humanities, Mathematics and Statistics, Science and Technology, Social Sciences, Workforce Development), que por sua vez se subdivem em outras. Para esse repositório, utilizou-se o mesmo procedimento de seleção dos objetos de aprendizagem que foi aplicado ao ROA LUME, pois estes repositórios posuem características semelhantes, sendo analisados nove objetos de aprendizagem (o primeiro recuperado de cada categoria principal).

Cabe destacar que o motor de busca do Merlot II permite que o usuário selecione sua língua nativa para consulta no repositório e também existe a possibilidade de construção de OA por meio da ferramenta ContentBuilder. Apresenta uma indição do que os outros usuários buscaram juntamente com o recurso localizado, como sugestão de visualização.

Nesse sentido, após a avaliação dos objetos de aprendizagem dos repositórios que adotam padrão LOM como base, realizou-se uma equivalência com os padrões MERLOT II, CESTAcore e OBBA, conforme pode ser observado no Quadro 4.

Quadro 4 - Equivalência entre os padrões LOM e suas derivações

\begin{tabular}{|c|c|c|c|c|}
\hline LOM & MERLOT II & CESTAcore & \multicolumn{2}{|c|}{ OBAA } \\
\hline general.title & Title & Titulo & obaa.general.title & Geral.Titulo \\
\hline general keyword & Keywords & Palavras-chave & obaa.general.keyword & Geral. "Palavras chave" \\
\hline general.description & Description & Descrição & obaa.general.description & Geral.Descrição \\
\hline $\begin{array}{l}\text { educational. } \\
\text { learningresourcetype }\end{array}$ & Material Type & $\begin{array}{l}\text { Recurso de } \\
\text { aprendizagem }\end{array}$ & $\begin{array}{l}\text { obaa.educational. } \\
\text { learningresourcetype }\end{array}$ & $\begin{array}{l}\text { Educacional "Tipo de } \\
\text { Recurso Educacional" }\end{array}$ \\
\hline technical.location & URL & Localização & obaa.technical location & $\begin{array}{l}\text { "Informaçōes Técnicas } \\
\text { Gerais Sobre o Objeto". } \\
\text { Localizaçăo }\end{array}$ \\
\hline $\begin{array}{l}\text { relation.resource } \\
\end{array}$ & & & obaa.relation.resource & Relação.Recurso \\
\hline general.coverage & & & obaa.general coverage & Geral Cobertura \\
\hline lifecycle.entity & Author & $\begin{array}{l}\begin{array}{l}\text { Entidades que } \\
\text { contribuiram }\end{array} \\
\end{array}$ & obaa.lifecycle.entity & "Ciclo de Vida". Entidade \\
\hline lifecycle.role & & Status & obaa. lifecycle.role & "Ciclo de Vida". Função \\
\hline lifecycle.contribute & Submitter & Tipo de contribuiçăo & obaa. lifecycle.Contribute & \begin{tabular}{|l|} 
"Ciclo de Vida". \\
Contribuição
\end{tabular} \\
\hline rights.description & Copyright & Direito Autoral & obaa.rights.description & Direitos.Descrição \\
\hline lifecycle.date & \begin{tabular}{|l} 
Date added \\
Date Modified
\end{tabular} & Data & obaa lifecycle date & "Ciclo de Vida". Data \\
\hline technical format & \begin{tabular}{|l} 
Technical \\
Format
\end{tabular} & Formato & obaa.technical format & Téenico.Formato \\
\hline general identifier & & Identificador & obaa.general.identifier & Geral.Identificador \\
\hline general language & Language & Idioma & obaa.general.language & Geral.Idioma \\
\hline
\end{tabular}

Fonte: Elaboração própria.

${ }^{1}$ Disponível em: <http://www.portalobaa.org/padrao-obaa/exemplos-de-oa-convertidos-para-o-obaa>. Acesso em: 22 mar. 2016. 
A partir da análise da amostra de metadados do acervo de objetos de aprendizagem dos repositórios investigados, apresenta-se o esquema de mapeamento relacional entre os padrões de metadados Dublin Core e LOM com suas respectivas equivalências com os demais padrões de metadados adotados pelos repositórios investigados, conforme pode ser visualizado no Quadro 5. O símbolo de equivalência $(\leftrightarrow)$ que aparece no Quadro 5 indica uma associação direta entre os elementos dos repositórios que adotam o padrão de metadados LOM.

Com a proposição do mapeamento relacional entre os padrões de metadados Dublin Core e LOM é possível melhorar a interoperabilidade entre repositórios de objetos de aprendizagem heterogênos e distribuídos para garantir um melhor refinamento nas buscas realizadas nesses ambientes.

Portanto, a partir do modelo de mapeamento relacional recomendado será proposto como trabalho futuro um modelo de indexação automática baseado em diferentes padrões de metadados educacionais para possibilitar uma recuperação de objetos de aprendizagem mais relevante.

Quadro 5 - Mapeamento relacional entre os padrões de metadados educacionais DC e LOM

\begin{tabular}{|c|c|c|c|}
\hline DC & DC - Refinamentos & LOM & LOM - Refinamentos \\
\hline dc.title & dc.title.altemative & general.title & $\begin{array}{l}\text { obaa.general.title } \leftrightarrow \text { Title } \\
\text { Geral.Título } \leftrightarrow \text { Titulo }\end{array}$ \\
\hline dc.subject & $\begin{array}{l}\text { dc.subject.keyword } \\
\text { dc.subject.category }\end{array}$ & general.keyword & $\begin{array}{l}\text { obaa.general.keyword } \leftrightarrow \text { Keywords } \\
\text { Geral."Palavras chave" } \leftrightarrow \text { Palavras-chave }\end{array}$ \\
\hline dc.description & $\begin{array}{l}\text { dc.description.abstract } \\
\text { dc.description.origem } \\
\text { dc.description.origin } \\
\text { dc.description.vinculoufrgs } \\
\end{array}$ & general.description & $\begin{array}{l}\text { obaa.general.description } \leftrightarrow \text { Description } \\
\text { Geral.Descrição } \leftrightarrow \text { Descrição }\end{array}$ \\
\hline dc.type & $\begin{array}{l}\text { dc.type.qualificationlevel } \\
\text { dc.type.qualificationname } \\
\text { dc.type.work }\end{array}$ & $\begin{array}{l}\text { educational.learningresource } \\
\text { type }\end{array}$ & $\begin{array}{l}\text { obaa.educational.learningresourcetype } \leftrightarrow \text { Material Type } \\
\text { Educacional."Tipo de recurso Educacional" } \leftrightarrow \text { Recurso de } \\
\text { aprendizagem }\end{array}$ \\
\hline dc.source & & technicallocation & $\begin{array}{l}\text { obaa.technical.location } \leftrightarrow \text { "Informações Técnicas Gerais Sobre o } \\
\text { Objeto". Localização } \leftrightarrow \text { URL }\end{array}$ \\
\hline dc.relation & $\begin{array}{l}\text { dc.relation.isreferencedby } \\
\text { dc.relation.requires }\end{array}$ & relation.resource & obaa.relation.resource $\leftrightarrow$ Relação.Recurso \\
\hline dc.coverage & $\begin{array}{l}\text { dc.coverage.culture } \\
\text { dc.coverage.temporal }\end{array}$ & general coverage & obaa.general.coverage $\leftrightarrow$ Geral.Cobertura \\
\hline dc.creator & & lifecycle entity & $\begin{array}{l}\text { obaa.lifecycle.entity } \\
\text { "Ciclo de Vida". Entidade } \leftrightarrow \text { Entidades que contribuiram } \\
\text { Author }\end{array}$ \\
\hline dc.publisher & dc.publisher.place & lifecycle.role & $\begin{array}{l}\text { obaa.lifecycle.role } \leftrightarrow \text { "Ciclo de Vida".Função } \\
\text { Status }\end{array}$ \\
\hline dc.contributor & $\begin{array}{l}\text { dc.contributor.advisor } \\
\text { dc.contributor.author } \\
\text { dc.contributor.role } \\
\text { dc.contributor.technicaldirector }\end{array}$ & lifecycle.contribute & $\begin{array}{l}\text { obaa.lifecycle.contribute } \leftrightarrow \text { "Ciclo de Vida". Contribuição } \\
\text { Submitter } \\
\text { Tipo de Contribuição }\end{array}$ \\
\hline dc.rights & dc.rights license & rights description & $\begin{array}{l}\text { obaa.rights.description } \leftrightarrow \text { Direitos. Descrição } \\
\text { Copyright } \leftrightarrow \text { Direito Autoral } \\
\end{array}$ \\
\hline dc.date & $\begin{array}{l}\text { dc.date.accessioned } \\
\text { dc.date.available } \\
\text { dc.date.created } \\
\text { dc.date.issued } \\
\text { dc.date.submitted } \\
\end{array}$ & lifecycle.date & $\begin{array}{l}\text { obaa.lifecycle.date } \leftrightarrow \text { “Ciclo de Vida".Data } \leftrightarrow \text { Data } \\
\text { Date Added } \\
\text { Date Modified }\end{array}$ \\
\hline dc.format & $\begin{array}{l}\text { dc.format.duration } \\
\text { dc.format.extent } \\
\text { dc.format.original } \\
\text { dc.format.support }\end{array}$ & technical format & $\begin{array}{l}\text { obaa.technical.format } \leftrightarrow \text { Technical Format } \leftrightarrow \text { Técnico.Formato } \\
\leftrightarrow \text { Formato }\end{array}$ \\
\hline dc.identifier & $\begin{array}{l}\text { dc.identifier.doi } \\
\text { dc.identifier.uri }\end{array}$ & general.identifier & obaa.general.identifier $\leftrightarrow$ Geral.Identificador $\leftrightarrow$ Identificador \\
\hline dc.language & dc.language.iso & general.language & obaa.general.language $\leftrightarrow$ Language $\leftrightarrow$ Geral.Idioma $\leftrightarrow$ Idioma \\
\hline
\end{tabular}

Fonte: Elaboração própria. 


\section{Considerações finais}

Os repositórios de objetos de aprendizagem são importantes ambientes digitais que têm como propósito o armazenamento de recursos educacionais. Os repositórios investigados forneceram os subsídios necessários para deduzir que os objetos de aprendizagem podem ser melhor recuperados quando estão descritos por meio um padrão de metadados educacional, pois dessa forma a expressividade desses recursos é consideravelmente aumentada.

$\mathrm{Na}$ investigação realizada, percebeu-se que as iniciativas nacionais são mais criteriosas na catalogação de seus recursos informacionais, pois utilizam um número maior de elementos do esquema de metadados para a catalogação dos objetos de aprendizagem.

O modelo de mapeamento apresentado entre os padrões de metadados Dublin Core e LOM sugere que é possível melhorar a interperabilidade entre objetos de aprendizagem mantidos por diferentes repositórios. A proposta de mapeamento relacional resultante da análise dos elementos dos esquemas de metadados permitiu estabelecer uma relação com todos os elementos do padrão Dublin Core o que possibilita determinar um modelo de indexação automática para diferentes repositórios de objetos de aprendizagem.

Dessa forma, conclui-se que foi possível estabelecer um modelo de correlação entre os padrões de metadados com um número significativo de elementos de cada esquema para facilitar o acesso aos objetos de aprendizagem e colaborar com o processo de indexação automática e, consequentemente aprimorar o processo de busca e recuperação de informação nos repositórios de objetos de aprendizagem.

\section{Referências}

BERNERS-LEE, T.; HENDER, J.; LASSILA, O. The Semantic Web: a new form of Web content that is meaningful to computers will unleash a revolution of new possibilities. New York: Scientific American, 2001.

DUBLIN CORE METADATA INITIATIVE (DCMI). 2016. Disponível em: <http://dublincore.org/>. Acesso em: 11 fev. 2016.

FERNEDA, E. Introdução aos Modelos Computacionais de Recuperação de Informação. Rio de Janeiro: Ciência Moderna, 2012.

GIL LeIVA, I.; FUJITA, M. S. L. (Org.). Política de indexação. São Paulo: Cultura Acadêmica; Oficina Universitária, 2012. v. 1.

LEARNING TECHNOLOGY STANDARDS COMMITTEE LEARNING OBJECT METADATA (IEEE LTSC LOM). IEEE Learning Technology Standards Committee (LTSC): standard for Learning Object Metadata (LOM). 2002. Disponível em: <http://grouper.ieee.org/groups/Itsc/wg12/files/LOM_1484_12_1_v1_Fin al_Draft.pdf>. Acesso em: 25 ago. 2015. 
LÓPEZ GUZMÁN, C. Los repositorios de objetos de aprendizaje como soporte a un entorno e-learning. 2005. 142f. Tese (Doutorado) Universidad de Salamanca, Salamanca, 2005.

LORIST, H. H. J.; van der MEER, K. Standards for digital libraries and archives: digital longevity. 2001. Disponível em: <https://static.aminer.org/pdf/PDF/000/512/466/standards_for_digital_li braries_and_archives_digital_longevity.pdf>. Acesso em: 20 dez. 2015.

MEADOW, C. T. et al. Text information retrieval systems. $3^{\text {rd }}$. ed. London UK: Elsevier, 2007.

NATIONAL INFORMATION STANDARDS ORGANIZATION (NISO). Understanding Metadata. Bethesda, MD: NISO Press, 2004. Disponível em:

<http://www.niso.org/standards/resources/UnderstandingMetadata.pdf>. Acesso em: 25 abr. 2016.

REHAK, D. R.; MASON, R. Engaging with the Learning Object Economy. In: LITTLEJOHN, A. (Ed.). Reusing online resources: a sustainable approach to e-learning. London: Kogan Page, 2003. p. 22-30.

SANTOS, P. L. V. A. C.; ALVES, R. C. V. Metadados e Web Semântica para estruturação da Web 2.0 e Web 3.0. DataGramaZero - Revista de Ciência da Informação, v. 10, n. 6, dez. 2009.

SILVA, D. L.; SOUZA, R. R. Representação de documentos multimídia: dos metadados às anotações semânticas. In: ENCONTRO NACIONAL DE PESQUISA EM CIÊNCIA DA INFORMAÇÃO (ENANCIB), 14., 2013. Florianópolis: ANCIB, 2013. p. 1-22.

SOUZA, M. I. F.; VENDRUSCULO, L. G.; MELO, G. C. Metadados para a descrição de recursos de informação eletrônica: utilização do padrão Dublin Core. Ciência da Informação, Brasília, DF, v. 29, n. 1, p. 93-102, abr. 2000.

TAROUCO, L. M. R.; RODRIGUES, A. P.; SCHMITT, M. A. R. Integração do MOODLE com repositórios abertos. Perspect. Ciênc. Inf., Belo Horizonte, v. 18, n. 1, p. 66-85, mar. 2013.

VICCARI, R. et al. Projeto OBAA: Relatório Técnico RTOBAA01 - Proposta de Padrão para Metadados de Objetos de Aprendizagem Multiplataforma. Porto Alegre: UFRGS/CINTED, 2009.

VICCARI, R. M. et al. Proposta de Padrão de Objetos de Aprendizagem Baseados em Agentes (OBAA). Revista Renote, v. 8, n. 2, 2010.

WELLER, M.; PEGLER, C.; MASON, R. Putting the pieces together: what working with learning objects means for the educator. Edinburgh: Elearn International, 2003. 